s'écoule dans une cuve pleine d'eau dans laquelle plonge l'extrémité de la conduite. Les vapeurs non condensées se rendent par une autre conduite dans une chambre de condensation.

Pour une partie de mercure contenu dans le minerai, il faut additionner ce dernier de deux parties de chaux. Lorsque le minerai est pyriteux, il faut augmenter la quantité de chaux. $\mathrm{Si}$ la proportion de chaux n'est pas assez élevée, il distille du sulfure de mercure avec le mercure; et lorsqu'on chauffe du minerai seul, sans chaux, la totalité du sulfure de mercure distille. On pourrait ainsi, si on le voulait, extraire du minerai à l'état du sulfure, la totalité de ce composé.

La dépense d'énergie dépend de la teneur du minerai en mercure et de la nature de sa gangue.

Ce procédé est applicable même aux minerais pauvres. Ces derniers pourraient être passés directement au four après pulvérisation et addition de chaux ou, ce qui vaudrait mieux, être coumis préalablement à une concentration.

Les avantages du four électrique nous paraissent tels que nous estimons que beaucoup de minerais pourraient supporter les frais de concentration. Cette dernière opération diminuerait forcément de beaucoup la grandeur de l'installation électrochimique nécessaire, la dépense d'énergie, etc.

(Journal de l'Electrolyse).

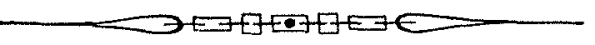

\section{Dispositif permettant l'utilisation des chutes d'eau dans les rivières navigables à berges inaccessibles}

Les chutes d'eau créées par les barrages sur les rivières navigables, du type de la Seine, de l'Yonne, de la Saône, par exemple, sont généralement inutilisées et inutilisables à cause des grandes difficultés que présente l'installation d'une usine hydraulique sur les berges encombrées, d'un côté par des sas d'écluse et autres ouvrages du service de la navigation, de l'autre côté par la culée servant de point d'appui au barrage.

L'établissement d'un canal d'amenée ou de fuite dans le voisinage immédiat de ce dernier ouvrage pourrait en compromettre la solidité, et, pour cette raison, le service de la navigation ne peut l'autoriser - à moins que ce canal ne passe très loin de la berge - Mais alors les travaux de dérivation qu'il faudrait exécuter pour utiliser la chute entraîneraient des dépenses hors de proportion avec l'énergie à mettre à profit et rendraient son prix de revient peu intéressant.

D'autre part, la renaissance de la navigation fluviale fait prévon que les travaux de doublement des sas déjà commencés en maints endroits seront poursuivis et généralisés sans arrêt. Il en résulte que l'existence d'une usine hydraulique établie en terre ferme, à l'emplacement que devra plus tard. occuper le deuxième sas serait des plus éphémères. Les frais d'amortissement, en présence de cette éventualité, devraient être majorés dans une proportion! telle que toute exploitation deviendrait impossible au point de vue financier.

Ces difficultés font donc que ces chutes restent inutilisées. Or, l'ênergie ainsi perdue représente une puissance très importante. Elle : pourrait être, grâce au trảnsport électrique de l'énergie; affectée utilement à diverses applications dans un certain rayon autour des barrages, voir même employée au halage électrique sur bien des rivières, si un moyen pratique existait pour capter cette énergie.

C'est ce moyen que propose $\mathrm{M}$. Henri CHANoIT, ingénieur des 'Arts et Manufactures. Il nous a paru que la description des dispositif $\tilde{f}^{\prime}$ mécaniques qu'il a brevetés dans le but de tirer parti de cette source de richesses jusqu'ici perdues, devaient être signalés aux lecteurs de La Houille Blanche.

Le plus grand nombre des chutes qui nous occupent contportent, comme l'indique le schéma d'installation de la figure ci-jointe : $\mathrm{I}^{\circ}$ un sas d'écluse du côté du chemin de halage; $2^{\circ}$ du côté du chemin de contre-halage une culée en maçonnerie très importante et servant de point d'appui aux fermettes mobiles du barrage proprement dit ; $3^{\circ}$ une pile centrale en maçonnerie partageant ce barrage en deux parties et servant aussi de point d'appu aux fermettes métalliques sur lesquelles 11 s'appuie.

Le sas d'écluse d'un côté, et la culée en maçonnerie de l'autre, s'opposent à l'aménagement économique sur la terre ferme d'une usine hydraulique avec ses canaux, chambres d'eau et turbines. Mais rien n'empêche l'installation de cette usine sur un bac amarré à l'aval de la pile centrale du barrage où elle ne peut en rien gêner le service de la navigation. Tel est le principe essentiel de l'usine hydraulique flottante imaginée par M. H. Chanoit.

Les figures ci-jointes permettent de se rendre compte au premier coup d'œil de quelle façon l'auteur réalise cette ingénieuse idée. Par dessus la pile centrale un siphón en tôle, supporté par des piles en treillis métallique, à hauteur convenable pour ne pas gêner le passage des. agents préposés au service de la navigation, amène l'eau de l'amont du barrage, à l'usine flottante proprement dite solidement amarrée à l'arrière de la pile centrale. Cette usine est constituée par un bac en tôle contenant une chambre d'eau, les turbines et les dynamos génératrices qu'elles actionnent, une petite pompe à air pour l'amorçage du siphon et enfin un petit logement pour le mécanicien. Un câble électrique aérien relie cette usine flottante à l'une des berges où, à cet effet, se trouve établi un support métallique suffisamment élevé pour que la présence de ce câble ne puisse en rien gêner le service de la batellerie.

La mise en service de cette usine est des plus simples :

Après l'avoir amarrée solidement à l'avant à des organaux scellés dans la pile centrale et à l'arrière à des ancreś mouillés à droite et à gauche, de façon à ce que l'axe de la tubulure de la chambre d'eau se trouve exactement dans le prolongement de l'axe de la grande branche du siphon, on met en place la partie mobile de cette branche qui se raccorde à la partie fixe par un point visible sur le dessin ; cette opération s'effectue facilement à l'aide de boulons de rappel convenablement disposés à cet effet. A ce moment la chambre d'eau est pleine jusqu'au niveau de l'eau à l'extérieur du bac.

On ferme ensuite le vannage des turbines et l'on procède à l'amorçage du siphon adducteur en faisant fonctionner la pompe à air mue par un petit moteur à essence. .Cette pompe est raccordée par une tuyaruterie flexible à la partie la plus haute du siphon. Dès que l'amorçage est terminé l'eau s'élève dans la chambre de mise en charge au niveau de l'eau en amont du barrage; les vannages des turbines étant alors ouverts, tout se passe comme dans une installation faite sur la terre ferme.

La disposition de la granide branche du siphon rendue complètement indépendante du bac flottant a pour but de permettre à celui-ci de suivre librement-les variations du niveau d'aval. En outre, en cas de crues dangereuses, il suffit de lâcher les boulons du joint réunissant les deux branches du siphon pour que la partie plongeante dans la chambre de mise en charge, libérée de ses attaches avec la partie fixe, vienne reposer sur uni siège établi à cet effet au fond de la chambre d'eau. L'usine flottante peut alors, en trente minutes environ, être amenée sur la rive où elle reste amarrée jusqu'à ce que la crue soit terminée.

Pour se rendre compte des frais d'installation d'une usine établie suivant ce système, il est nécessaire de recourir à un exemple. L'inventeur suppose dans ce but qu'il s'agisse d'utiliser l'énergie de la chute du barrage d'Albon (Seine-et-Oise) où, sans nuire au service de la navigation, l'on peut utiliser un minimum de 7000 litres sous une dénivellation variant de o à 2 mètres.

La dépense qu'entraînerait l'installation d'une usine flot' tante capable.de transformer cette puissance en énergie élec- 
trique serait, d'après un devis que nous avons sous les yeux : $\mathrm{Bac}$ flottant, siphon, agrès et aménagements. . fr. 38700 Turbines, vannages, transmissions et accessoires.... 42500 Machines dynamor génératrices et appareillage élec-

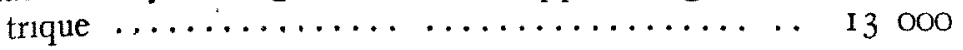

Total des frais d'établissement de l'usine.. fr. 94000

Voici maintenant comment sont estimés les frais de toute nature que nécessite son fonctionnement :

Personnel (mécaniciens, électriciens) et assurance fr. Assurance du matériel, graissage, impositions..... Réparations et annuité d'amortissement en 20 ans.. Frais généraux et redevance à l'Etat............

Total des dépenses annuelles de fonctionnement.................... fr.

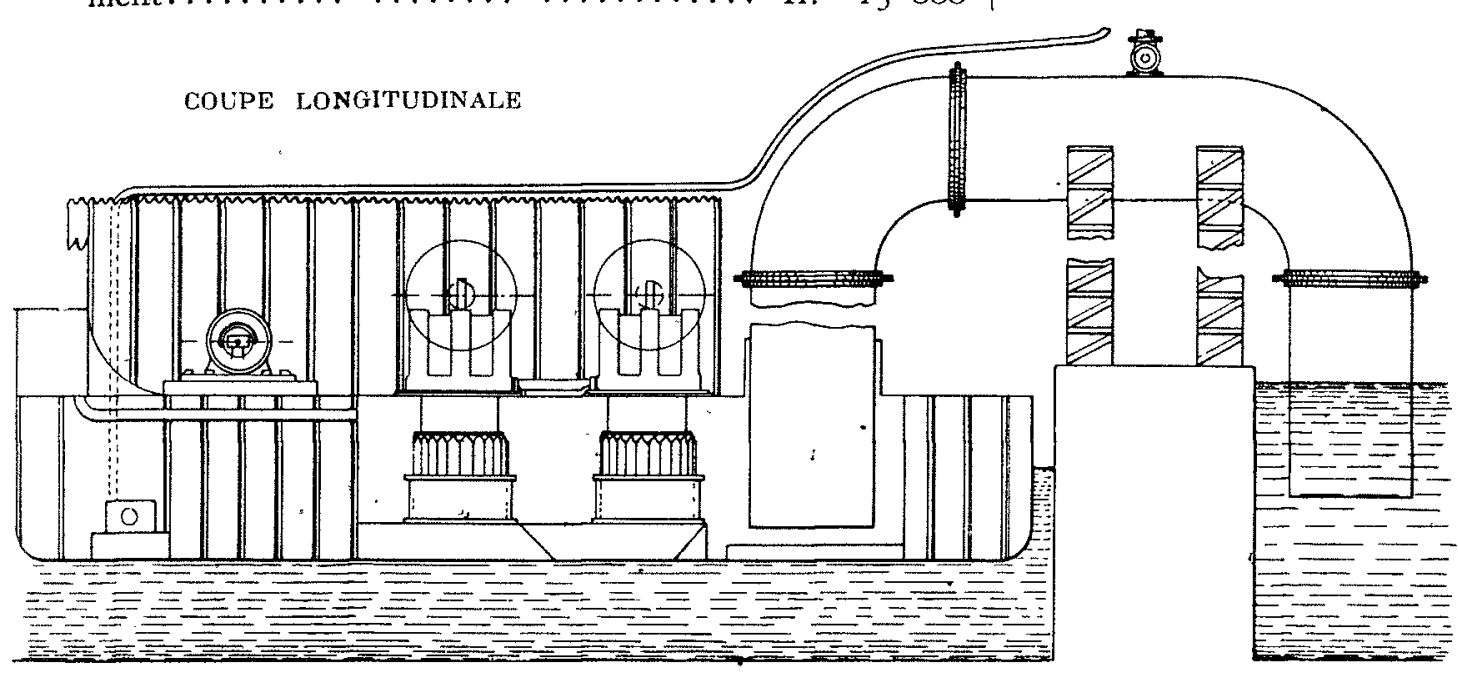

5200

2

du cheval-heure effectif ressortirait alors à o fr. or $8 \mathrm{I}$. Soit, en chiffres ronds, moins de deux centimes par cheval-heure.

Pour tenir compte des frais d'établissement d'une ligne de transport électrıque de l'énergie devant permettre de l'utiliser à une certaine distance de l'usine, M. Chanoit suppose les frais de premier établissement de cette dernière augmentés de $50 \%$. Le total s'en éleverait ainsi à I $4 \mathrm{I} 000$ francs. Les frais d'exploitation vont se trouver de ce chef portés à 20 I 40 francs. D'autre part, en admettant une perte de $25 \%$ dans la ligne de transport d'énergie il ne reste plus à son extrémité que 621000 chevaux-heure disponibles coûtant 20 I40 francs par an, ils mettent le prix de revient chez le consommateur à $O$ fr. 0322 . Cette dépense serast ainsi inférieure ou au plus égale à celle du petit moteur à gaz, à pétrole ou à vapeur qui, suivant les circonstances locales, varie de o fr. 05 à 0 fr. 25 le cheval-heure.
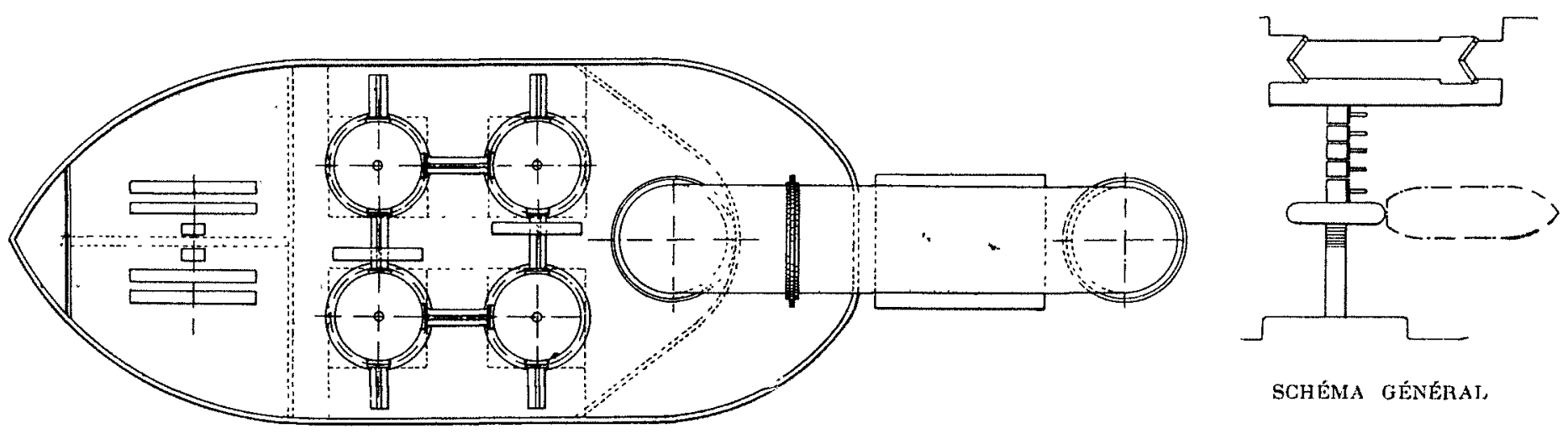

VUE EN PLAN

A combien cela met-il le prix de revient du cheval ? Voici à ce sujet le calcul que fait l'auteur du projet :

Les chutes qui nous intéressent ne sont pas de hauteur constante mais varient suivant les époques de l'année. Or, la statistique indique qu'en ne tenant pas compte des chutes inférieures à $\mathrm{O}^{\mathrm{m}} 8 \mathrm{o}$, le nombre total de chevaux-heure utilisables annuel lement dans un barrage donné est de 6500 fois la puissance maximum horaire constatée. Dans le cas du barrage d'Albon pris comme exemple, où la puissance maximum qu'on peut réaliser avec le débit autorisé de 7000 litres est de 182 chevaux, le nombre total de chevaux-heure utilisables dans une année serait de $6500 \times 182=1$ I 18000 ou de 945000 sur l'arbre des turbines si l'on admet pour celles-ci un rendement de 80 pour Ioo (un peu fort selon nous).

L'auteur admettant d'autre part que le rendement des dynamos est de 88 pour Ioo, il en résulte que l'énergie électrique annuellement disponible aux bornes des dynamos génératrices installées à bord de l'usine flottante serait de 830000 chevaux-heure.

Les frais d'exploitation s'élevant à 15000 francs, le prix
On pourrait sans doute faire un certain nombre d'objections à ce système, mais nous n'en voulons formuler aucune car tel inconviénient qui sur le papier semble un obstacle s'évanouit souvent après ure expérience intelligemment faite alors qu'au contraire des difficultés auxquelles on ne songe point dans l'étude d'un projet constituent des empêchements sérieux à la mise en pratique. Nous ne savons pas si l'usine hydro-électrique flottante a été, ou va être appliquée quelque part. Mais, dans tous les cas, elle mérite de l'être. ILes plans et les devis qui nous ont été soumis montrent qu'elle peut dans la généralité des circonstances, convenir à l'utilisation de l'énergie perdue sur les barrages et cela dans des conditions de prix de revient très acceptables.

L'application de ce système dont l'ingéniosité n'a d'égale que la simplicité, aurait donc l'avantage de permettre l'utilisation d'une source de richesse naturelle jusqu'ici inexploitée; l'Etat et les particuliers pourraient en profiter sans qu'il en résultât aucun inconvénient pour le service de la navigation, le volume d'eau à utiliser étant fixé par le service même des Ponts et Chaussées pour chaque cas particulier. Il présenterait au surplus sur les installations hydro-électriques ordinaires d'importance comparable, cet avantage qui n'est pas à 
dédaigner : avant de commencer les travaux d'une semblable installation, il serait possible d'en fixer à quelques francs près la dépense, - d'où la fixation assez certaine du prix de revient de l'énergie - calcul qui est, comme le savent tous les ingénieurs, d'une exactitude généralement très problématique quand il s'agit de barrages, canaux et usine à établir sur un terrain qui offre des surprises et pour utiliser un débit qui en réserve encore de plus désagréables.

Cette initiative vaut qu'on l'encourage; puissions-nous avorr contribué à porter l'attention sur elle.

E. C.

\section{L'Électricité et la Police correctionnelle}

\section{COUR D'APPEI DE NANCY \\ Audience du 13 juillet 1904.}

Présidence de $M$. DE Luxer.

CONDAMNATION POUR VOL D'ÉLECTRICITÉ.

L'électricité est susceptible d'une propriété privée par l'accumulation qui en est faite, la direction qu'on lui donne, l'usage auquel on l'emploie: force, chaleur ou lumière $\left(^{*}\right)$.

Par suite, commet un vol celui qui, pour faire fonctionner des lampes, se sert du courant transmis par une usine électrique, en l'empêchant de passer par le compteur.

(Viller contre fabius Henrion).

La Gour,

Attendu qu'il résulte, de l'information et des débats, la preuve que, le I4 mars I 904 , le monteur Michel, attaché à l'usine électrique de Fabius Henrion, à Pagny-sur-Moselle, s'étant rendu chez le prévenu Viller, abonné à l'usine, pour y vérifier son compteur, remarqua que les deux bornes saillantes de l'appareil étaient réunies par deux fils et que, grâce à cette disposition, ajoutée subrepticement, le courant électrique arrivait directement aux lampes sans passer par le compteur, qui se trouvait hors circuit et marquait le chiffre de 1721 .

Attendu que, le 22 mars suivant, le même monteur, se rendant de nouveau chez Viller, constata avec le contremaitre Rougelowski, qui l'assistait, que le compteur était toujours hors circuit et yu'il continuait à indiquer le chiffre de $172 \mathrm{I}$, bien que, dans l'intervalle des deux visites, les lampes eussent été allumées; qu'en ayant fait l'observation à Viller, celui-ci monta vivemement sur son établi et sépara les fils en s'écriant: "Vous pouvez certifier l'avoir vu, moi, je nierai ";

Artendu que, le lendemain, lorsque la gendarmerie procéda à son enquête, le compteur indiquait i 726 , ce qui démontre, d'une part, que la consommation quotidienne de Viller pouvait être évaluée à environ 5 hectowatts;

Attendu que l'attitude de Viller, lors de la visite des employés de Fabius Henrion, son empressement à rétablir la communication, les paroles qu'il a prononcées à ce moment établissent jusquà la dernière évidence que lui seul, quoiqu'il prétende le contraire, est l'auteur de la mise hors circuit du compteur; que le moyen mis en pratique pour y arriver dénote, d'ailleurs, une certaine habileté professionnelle de la part de celui qui y a eu recours; que cette habileté se rencontre précisément chez Viller, qui a aidé le contremaître Rougelowski dans ses installations électriques, et, par suite, acquis des connaissances pratiques qui lui ont servi pour la réalisation de la combinaison fraudu-

(*) Cette théorie n'est pas admise dans tous les pays étrangers. La jurisprudence allemande s'est prononcée en sens contraire. Le Tribunal de l'Empire, par arrêt du 20 octobre 1896 , a admis que dans la définition du vol, le mot choses s'entendait seulement des objets corporéls. Or, d'après les dernières données de la science, l'électricité ne serait pas un fluide, un courant circulant le long-d'un fil, mais une énergie résidant dans le corps électrisé et résultant d'une vibration particulière de ce corps. Devant cette jurisprudence, le législateur allemand a dû, pour ne pas laisser impuni le détournement d'énergie électrique, en faire un délit particulier puni par une loi du 9 avril 1900 . Il existe de même une loi spéciale en Angleterre'(loi du 18 août I 882 ). leuse à laquelle il s'est livré dans le but de s'approprier la chose d'autrui ;

Attendu, en effet, que quel que soit l'aspect sous lequel l'électricité doive être envisagée, qu'on la considère comme un fluide ou une énergie, il est incontestable qu'elle est susceptible d'une propriété privée par l'accumulation qui en est faite, la direction qu'on lui donne, l'usage auquel on l'emploie, force, chaleur ou lumière; qu'en consommant donc sans la payer, puisque le compteur par lequel elle ne passait plus n'enregistrait pas son débit, une certaine quantité d'électricité, Viller a ainsi fait mainmise, à l'insu de son propriétaire Fabius Henrion, et contre son gré, sur une chose qui ne lui appartenait pas et se l'est appropriée frauduleusement ;

Attendu qu'on objecterait vainement qu'en l'éspèce il ne peut y avoir appréhension frauduleuse, puisque l'électricité était remise volontairement par Fabius Henrion à Viller; que cette remise, tout d'abord, était subordonnée à la condition que l'électricité passât par le compteur ; qu'il est certain, d'autre part, que Fabius Henrion gardait jusqu'au compteur la propriété exclusive de l'électricité qu'il avait produite et que c'est seulement lorsqu'elle avait franchi l'appareil, après y avoir été enregistrée, qu'elle était remise à Viller; qu'en la soustrayant donc avant son passage par le compteur, et ce, à l'aide de moyens frauduleux, le prévenu s'est rendu, par suite, coupable du délit de vol retenu à sa charge par les premiers juges dont il échet de confirmer la décision, tant surla déclaration de culpabilité que sur la peine prononcée et les dommages-intérêts alloués à la partie civile;

Par ces motifs,

Reçoit en la forme l'appel de Viller envers le jugement du Tribunal de Nancy du 7 mai 1904 ;

Au fond:

Déclare ledit appel mal fondé, le rejette ;

Confirme, en conséquence, le jugement attaqué dans toutes ses dispositions:

Condamne Fabius Henrion, partie civile, à tous les dépenș de première instance et d'appel, sauf son recours contre. Viller, qui devra les supporter en définitive;

Fixe au minimum contre Viller la durée de la contrainte par corps, s'il y a lieu de l'exercer.

\section{TRIBUINAI CIVII DE PAU}

Audience du 10 décembre 1904.

Présidence de M. Dubois de Lhermont, président.

RESPONSABILITÉ DES ACCIDENTS PAR I'ÉLECTRICITÉ.

I. Tout propriétaire d'un bâtiment est responsable des défauts qui $y$ sont inhérents. Or, il faut assimiler aux batiments les choses qui en dépendent par incorporation, tels que les mécanismes et les appareils.

II. Le danger d'une installation électrique étant connu, toutes les précautions possibles doivent être prises pour $y$ parer.'

III. Lorsqu'un enfant est victime d'un accident mortel, les Tribunaux doivent, pour fixer la réparation due aux parents, apprécier, avec le dommage moral, le dommage que leur caise la perte du salaire de l'enfant et l'ássistance qu'ils devaient én espérer pour l'avenir.

\section{(S... contre Société Electrioue.)}

Ainsi jugé par la décision suivante:

Le Tribunal,

$\mathrm{Vu}$ l'assignation du 30 juin 1904 .

Attendu qu'il est constant que le 28 avril igo4, le jeune $S_{4}$ âgé de moins de I 4 ans, apprenti jockey chez X..., a été viçtim d'un accident mortel; qu'il a été foudroyé en touchant lêt câble conducteur d'un courant électrique à haute tension ;

Attendu qu'il a été expliqué que la Société électrique dés Pyrénées, pour distribuer la lumière, a établi dans divèl endroits des appareils dits transformateurs, qui: reçoiven l'énergie électrique venant de l'usine centrale, la transforment et la conduisent dans les différents immeubles d'un même quar: tier au moyen des fils s'amorçant sur le câble du transforma teur; que l'éclairage électrique a été-installé depuis plusieurs années dans la propriété de X... par les soins de la Sóciéte électrique ; que le transformateur. a d'abord été placé aưdestuts des écuries, les-fils à hate-tensión passant au-dessus-des 